\title{
In Situ ATR FTIR Monitoring of the Formation of Functionalized Mono- and Multilayers on Germanium Substrate: from 7-Octenyltrichlorosilane to 7-Carboxylsilane
}

\author{
Jasmina Matijašević, Norbert Hassler, Gerald Reiter, and Urs Peter Fringeli* \\ University of Vienna, Institute of Biophysical Chemistry, Althanstrasse 14, 1090 Vienna, Austria
}

Received October 1, 2007. In Final Form: November 22, 2007

\begin{abstract}
The development and optimization of biomimetic surfaces required for biosensors and medical assays are made more efficient by quantitatively monitoring the surface chemical reactions in situ by means of attenuated total reflection (ATR) FTIR spectroscopy. single-beam-sample-reference (SBSR) ATR, as well as modulated excitation (ME), techniques have been applied to get physicochemical information on growth and structure of the surface layer. SBSR and ME methods result in optimum background compensation and signal-to-noise ratio. Surface modification was performed on a germanium multiple internal reflection element (Ge-MIRE). Activation of the surface resulted in free $\mathrm{Ge}-\mathrm{OH}$ groups used for a spontaneous chemical reaction with 7-octenyltrichlorosilane (7-OTCS) in toluene. Formation of $\mathrm{Ge}-\mathrm{O}-\mathrm{Si}$ bonds was enabled by hydrolization of $\mathrm{Si}-\mathrm{Cl}_{3}$ after partial elimination of a tightly bound thin water layer covering the MIRE. Unwanted side-reaction by hydrolization of $\mathrm{Si}-\mathrm{Cl}_{3}$ in solution followed by polymerization paralleled this process. Steady growing of the silane layer to multilayer thickness with increasing time was observed in all experiments. Most unexpectedly, in some experiments the end-standing double bond of the silane layer was found to be partly oxidized even after being exposed only to toluene, probably because of catalysis by molecular sieve nanoparticles remaining in toluene after drying. Finally, theoretical means are presented enabling the calculation of the spectrum of dissolved 7-OTCS in toluene, a prerequisite for background compensation during in situ studies of the growing layer.
\end{abstract}

\section{Introduction}

Organized organic molecular monolayer and multilayer structures formed at solid surfaces by spontaneous adsorption/ reaction of molecules from solutions are known as self-assembled mono- and multilayers (SAMs). They have been studied extensively because they can form well-ordered, cross-linked two-dimensional structures which are important for biomaterials ${ }^{1}$ and biosensors, ${ }^{2}$ nonlinear optical devices, ${ }^{3,4}$ HPLC, ${ }^{5}$ nanolithography, ${ }^{6}$ a variety of heterogeneous phenomena including catalysis, ${ }^{7}$ corrosion, ${ }^{8}$ lubrication, ${ }^{9}$ adhesion, ${ }^{10}$ and dispersion and deagglomeration of nanoparticles. ${ }^{11}$ Such self-assembled structures may provide formation of molecular structures with known spacings, orientation, and thickness. ${ }^{12}$

* To whom correspondence should be addressed. E-mail: urs.peter.fringeli@univie.ac.at. Tel: +43-1-4277-52502.Fax: +43-1-42779525 .

(1) Vermette, P.; Gauvreau, V.; Pézolet, M.; Laroche, G. Colloids Surf, B: Biointerfaces 2003, 29, 285-295.

(2) Senaratne, W.; Andruzzi, L.; Ober, C. K. Biomacromolecules 2005, 6, 2427-2448.

(3) Li, D.; Ratner, M. A.; Marks, T. J. J. Am. Chem. Soc.1990, 112, 73897390.

(4) Swalen, J. D.; Allara, D. L.; Andrade, J. D. Chandross, E. A.; Garoff, S.; Israelachvili, J.; McCarthy, T. J.; Murray, R.; Pease, R. F.; Rabolt, J. F.; Wynne, K. J.; and Yu, H. Langmuir 1987, 3, 932-950.

(5) Ito, K.; Ariyoshi, Y.; Tanabiki, F.; Sunahara, H. Anal. Chem. 1991, 63 , $273-276$.

(6) Liu, S.; Maoz, R.; Schmict, G.; Sagib, J. Nanoletters 2002, 2, 10, 10551060.

(7) Durand, R. R.; Bencosme, S. C.; Collman, J. P.; Anson, F. C. J. Am. Chem. Soc. 1983, 105, 2710-2718.

(8) Li, G.; Ma, H.; Jiao, Y.; Chen, S. J. Serb. Chem. Soc. 2004, 69, 10, 791805.

(9) Green, J-B. D.; McDermott, M. T. and Porter, M. D.; Siperko, L. M. J. Phys. Chem. 1995, 99, 10960-10965.

(10) Porter, M. D.; Bright, T. B.; Allara, D. L. and Chidseyi, C. E. D. J. Am Chem. Soc. 1987, 109, 3559-3568.

(11) Stojanović, D. B.; Vuković, G. D.; Orlović, A. M.; Uskoković, P. S.; Aleksić, R. R.; Bibić. N. M.; Dramićanin, M. D. Chem. Ind. 2007, 61, 3, 109116.
SAMs are formed by using bifunctional molecules featuring usually a small head group with an affinity for the substrate combined with a hydrocarbon tail. ${ }^{3}$ One particular class of SAMs are derivatives of silanes, e.g., $n$-alkyltrichlorosilanes, which act as coupling agents between organic compounds (e.g., proteins) and inorganic hydroxylated surfaces (glass, quartz, aluminum, germanium, silicon, and metal oxides). The $\mathrm{SiCl}_{3}$ function of $n$-octenyltrichlorosilane ( $n$-OTCS) enables covalent attachment of the molecules at surfaces rich of hydroxyl groups, while the terminal ethylenic double bond provides a convenient path for the activation of the outer layer surface through oxidation. ${ }^{13,14}$ SAMs of $n$-OTCS have been studied using a wide variety of techniques, including ellipsometry and low-angle X-ray reflectivity, ${ }^{3,15}$ contact angle goniometry, ${ }^{16}$ electrochemical impedance spectroscopy (EIS), ${ }^{8}$ wettability and X-ray photoelectron spectroscopy (XPS), ${ }^{17}$ FTIR spectroscopy, ${ }^{18-21}$ surface-enhanced Raman scattering (SERS) and infrared reflection-absorption spectroscopy (IRRAS), ${ }^{22}$ and atomic force microscopy (AFM). ${ }^{23}$

(12) Finklea, H. O.; Robinson, L. R.; Blackburn, A. and Richter, B. Langmuir 1986, 2, 239-244.

(13) Netzer, L. and Sagiv, J. J. Am. Chem. Soc. 1983, 105, 674-676.

(14) Zhang, Y.; Terrill, R. H.; Bohn, P. W. Chem. Mater. 1999, 11, $2191-$ 2198.

(15) Wasserman, S. R.; Whitesides, G. M.; Tidswell, I. M.; Ocko, B. M.; Pershan, P. S. and Axe, J. D. J. Am. Chem. Soc. 1989, 111, 5852-5861.

(16) Wu, B.; Mao, G.; Ng, K. Y. S. Colloids Surf., A: Physicochem. Eng. Aspects 1999, 162, 203-213.

(17) Wasserman, S. R.; Tao Yu-Tai, Whitesides, G. M. Langmuir 1989, 5 , 1074-1087.

(18) Netzer, L.; Isovici, R.; Sagiv, J. Thin Solid Films 1983, 100, 67

(19) Elmore, D. L.; Chase, D. B.; Liu, Y.; Rabolt, J. F. Vib. Spectrosc. 2004 $34,37-45$.

(20) Maoz, R.; Sagiv, J.; Degenhatdt, D.; Möhwald, H.; Quint, P. Supramol. Sci. 1995, 2, 9-24.

(21) Gun, J.; Isovici, R.; Sagiv, J. J. Colloid Interface Sci 1984, 101, 1, 201

(22) Leverette, C. L.; Dluhy, R. A. Colloids Surf., A: Physicochem. Eng. Aspects 2004, 243, 157-167.

(23) Nakagawa, T.; Ogawa, K.; Kurumizawa, T. Langmuir 1994, 10, 525529. 
We focus on an in situ study of the reaction of 7-octenyltrichlorosilane (7-OTCS) with the hydroxylated surface of a germanium (Ge) multiple internal reflection element (MIRE) by means of FTIR ATR spectroscopy. Since the 7-OTCS surface layer is planed as anchor for covalently bound biomimetic surfaces, we directed attention to a most accurate quantitative analysis of layer growth, surface concentration, and structure. Knowledge of structural details will be of utmost importance for the understanding of success and failure of any further surface chemical reaction step.

This analysis consists of the determination of basic quantities such as integrated molar absorption coefficients of relevant functional groups, time-resolved measurement of layer growth, and structural analysis of the layer assembly. Special attention had to be paid to background compensation, since 7-OTCS in solution spontaneously reacts with the Ge surface disabling the measurement of a reference spectrum reflecting only dissolved 7-OTCS in toluene. This problem was overcome by the application of basic ATR theory. ${ }^{24}$ As a final chemical step, the end-standing double bond of 7-OCTS was converted to a carboxylic acid group by oxidation with ozone resulting in 7-carboxylsilane.

\section{Materials and Methods}

2.1. Chemicals. 7-OTCS, $\mathrm{C}_{8} \mathrm{H}_{15} \mathrm{SiCl}_{3}$, (95\% contains $10-15 \%$ isomers) was purchased from $\mathrm{ABCR} \mathrm{GmbH \& Co}$. and used as received. Toluene (99.9\%) was purchased from Sigma Aldrich. 7-OTCS was handled in a dry nitrogen atmosphere to prevent hydroxylation by atmospheric humidity. Moreover, tubes leading to the ATR cell were preconditioned by a short flow of 7-OTCS solution directed to waste.

2.2. Spectra Acquisition. 2.2.1. FTIR-ATR Equipment. MIREs of trapezoidal shape were made of germanium (Ge, $52 \times 20 \times 2.1$ $\left.\mathrm{mm}^{3}\right)$ and cadmium telluride $\left(\mathrm{CdTe}, 52 \times 19.5 \times 1.6 \mathrm{~mm}^{3}\right)$. At the nominal angle of incidence $\theta=45^{\circ}$ one obtained 19.4 and 25.3 active internal reflections, respectively. Stationary and modulated excitation (ME) spectra were recorded with Bruker IFS 25 and IFS 66 spectrometers, at 15 and $22{ }^{\circ} \mathrm{C}$, respectively. Both instruments were equipped with a lift-model single-beam-sample-reference (SBSR) ATR mirror attachment with a hydrodynamically optimized and water-thermostated cell (flow-through cuvette) made of PEEK [poly(ether ether ketone)]. Spectra were recorded with parallel and perpendicular polarized IR light produced by an aluminum grid polarizer on a KRS-5 substrate. A mercury cadmium telluride (MCT) detector was used. Spectral resolution was $4 \mathrm{~cm}^{-1}$ with a zero filling factor of 4. The Blackman-Harris three-term function was used for apodization.

2.2.2. SBSR Method. The SBSR technique enables the conversion of a single-beam spectrometer into a pseudo-double-beam spectrometer (Figure 1). This is performed by optical conversion of the incoming focusing beam into a parallel beam of half-height of a MIRE (see Figure 1A). Sample (S) and reference (R) cuvettes may now be placed on the same MIRE. Change from $S$ to $R$ and vice versa is performed by means of a computer controlled lift (L) letting the IR beam to pass alternatively through sample and reference (see Figure 1B).

As $\mathrm{S}$ and $\mathrm{R}$ can be measured with a single beam, this technique is referred to as the single-beam-sample-reference (SBSR) method. The corresponding spectra, i.e., $\mathrm{S} / \mathrm{R}$ and $-\log (\mathrm{S} / \mathrm{R})$, are called $\mathrm{SBSR}$ transmittance and absorbance spectra, respectively.

The SBSR cell was made of PEEK consisting of independently accessible $S$ and $R$ cuvettes on both sides of the MIRE. Viton O-rings were used for sealing. The cuvettes have been hydrodynamically optimized with respect to flow-through featuring a rectangular shape $\left(35.35 \times 7.5 \mathrm{~mm}^{2}\right)$, terminated on either side by a half circle $(r=$ $3.75 \mathrm{~mm}$ ). The distance between the MIRE and cell body was $\sim 150$

(24) Fringeli, U. P.; Reiter, G.; Matijašević, J. A new experimental and theoretical approach to in situ monitoring of surface chemical reactions by FTIR ATR spectroscopy. Appl. Spectrosc., in preparation.
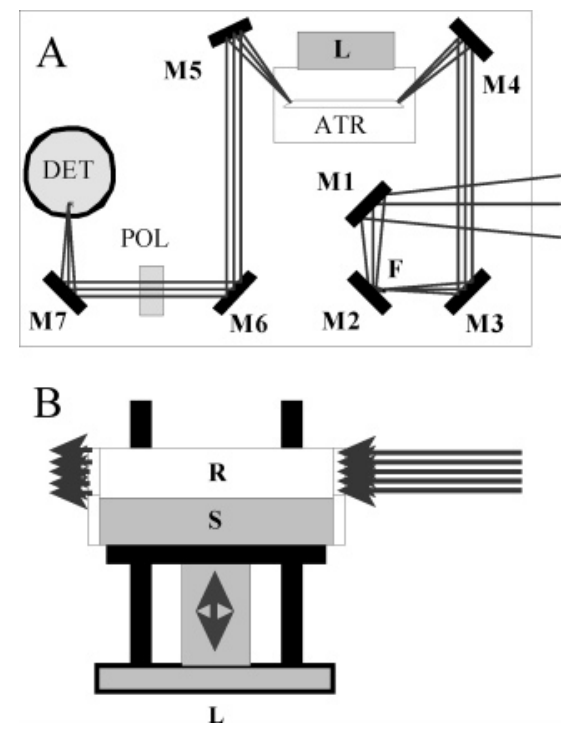

Figure 1. Single-beam-sample-reference (SBSR) ATR attachment. (A) The focus in the sample compartment of the spectrometer is displaced to the position F by the planar mirrors M1 and M2. The off-axis parabolic mirror $\mathrm{M} 3$ produces a parallel beam with a diameter of $1 \mathrm{~cm}$, i.e., half of the height of the MIRE. The cylindrical mirror M4 focuses the light to the entrance face of the MIRE. M5 which has the same shape as M4 reconverts to parallel light directing it via the planar mirror M6 through the polarizer POL and then being focused to the detector DET by the off-axis parabolic mirror M7. (B) Alternating change from sample $(\mathrm{S})$ to reference $(\mathrm{R})$ is performed by computer controlled lifting and lowering of the ATR cell body. Reproduced from ref 38 by permission.

$\mu \mathrm{m}$, resulting in a volume of $\sim 50 \mu \mathrm{L}$ per cuvette. Temperature regulation of the SBSR cell within $\pm 0.2{ }^{\circ} \mathrm{C}$ was achieved by an external thermostat with circulating water. The temperature of the cell was monitored throughout the experiment by means of a digital contact thermometer. All SBSR equipment was obtained from OPTISPEC. ${ }^{25}$

2.2.3. ME Experiments. Any sample responding reversibly to the change of an external parameter such as pressure $(p)$, temperature $(T)$, concentration $(c)$, electric field $(E)$, light flux $(F)$, etc. may be stimulated by a periodic change of the respective parameter. This method is referred to as modulated excitation spectroscopy (MES).

At the beginning of a ME experiment the system will relax from its equilibrium state to a new quasi-steady state. This will last about three times the largest relaxation time of the system. Now data acquisition may begin by measuring $N$ spectra at equidistant time slices within each repetitive stimulation period, $T$, thus approximating the continuous sample response $A(\tilde{v}, t)$. These $N$ so-called sample point spectra are then co-added and subjected to a digital phase sensitive detection (PSD) resulting in the so-called phase-resolved or demodulated spectra according to eq 1 .

$$
A_{k}^{\phi_{k} \mathrm{PSD}}(\tilde{v})=\frac{2}{T} \int_{0}^{T} A(\tilde{v}, t) \cdot \sin \left(k \omega t+\phi_{k}^{\mathrm{PSD}}\right) \mathrm{d} t
$$

$A(\tilde{v}, t)$ represents the time-resolved steady-state spectral response of a sample that is periodically stimulated at frequency $\omega$. In a general case, $A(\tilde{v}, t)$ may be a superposition of partial responses in the fundamental $\omega$ as well as in higher harmonics $k \times \omega$ according to eq 2

$$
A(\tilde{v}, t)=\sum_{k} A_{k 0}(\tilde{v}) \cdot \sin \left(k \cdot \omega \cdot t+\phi_{k}\right)
$$

(25) OPTISPEC, Rigistrasse 5, CH-8173 Neerach (Switzerland), www.optispec.ch. 
Introducing eq 2 into eq 1 results in

$$
A_{k}^{\phi_{\mathrm{R}} \mathrm{PSD}}(\tilde{v})=A_{k 0}(\tilde{v}, t) \cdot \cos \left(\phi_{k}-\phi_{k}^{\mathrm{PSD}}\right)
$$

Equations 1 and 3 explain the principal features of PSD. The periodic stationary sample response $A(\tilde{v}, t)$ has to be multiplied by a demodulation function. In the case of eq 1 , demodulation occurs at frequency $k \omega$. Moreover, a new arbitrary parameter, $\phi_{k}^{\mathrm{PSD}}$, is introduced. As a consequence, this procedure extracts the amplitude of the sample response with frequency $k \omega$ from the overall signal resulting in phase resolved spectra described by eq $3 . \phi_{k}$ denotes the phase delay in the $k \omega$ response between the onset of simulation with frequency $\omega$ and the response of the sample. In the case of harmonic stimulation with frequency $\omega$, a signal response at $k \neq 1$ unambiguously points to nonlinear steps in the reaction scheme of the system. MES has also access to time-resolved information, since phase delays, $\phi_{k}$, introduced by the system are related to the reaction scheme as well as to the underlying rate constants. If the period of stimulation is long compared to relaxation times of the system, e.g., as in case of a chemical stimulation, time-resolved information is no longer available; however, there remain still two most relevant advantages of MES over conventional difference spectroscopy. (i) The signal-to-noise ratio of a phase-resolved spectrum is at least 10 times better than achievable by conventional difference spectroscopy, since in an electronic sense, MES is a narrow band technique. (ii) MES with PSD results in a Fourier analysis of the sample response which is significant of the underlying reaction scheme. Conventional difference spectroscopy has no direct access to this information.

In this experiment the stimulation period was $T=5 \mathrm{~min}$, i.e., the layer was $2.5 \mathrm{~min}$ in contact with both stimulating solutions of $\mathrm{pH}$ 4 and 8, respectively. Six modulation cycles have been added leading to an overall accumulation time of $30 \mathrm{~min}$ per presented ME spectrum. For a detailed description of MES, the reader is referred to ref 26.

2.3. Sample Preparation. 2.3.1. Preparation of $\mathrm{CdTe}$ and $\mathrm{Ge}$ MIRE. The only reason for the use of CdTe MIRE was the enhanced spectral transmittance $4000-500 \mathrm{~cm}^{-1}$ enabling access to $\mathrm{Si}-\mathrm{Cl}$ vibrational bands. No surface chemical reactions were aimed with this material. For cleaning, the plate was manually polished on a Nylon cloth using $1 / 4 \mu \mathrm{m}$ diamond paste. Then, it was cleaned with ethanol, and after drying under a flow of air, it was mounted into the SBSR cell.

An activation procedure was applied only to the Ge MIRE in order to produce free hydroxyl groups at the surface which is a prerequisite for the reaction with chlorosilanes. The first step in this procedure was polishing of both sides of the plate by machine (Logitech PM5, 60rpm for $2 \mathrm{~min}$ ) using a non-woven polypropylene fabric immersed with $0.1 \mu \mathrm{m}$ diamond particle suspension ( $\mathrm{AB}$ Technics, Tribuswinkel, Austria) followed by cleaning with ethanol. Remaining organic impurities were then removed by plasma cleaning (Harrick Scientific Products, Inc., Pleasantville, NY) for $3 \mathrm{~min}$ in laboratory atmosphere at $(1.2 \pm 0.2) \times 10^{-2}$ mbar. The resulting $\mathrm{GeO}_{2}$ surface layer was activated by immersing the plate in ultrapure water for $30 \mathrm{~min}$ and drying it by a pure air flow. The MIRE was considered to be clean if the $v\left(\mathrm{CH}_{2}\right)$ bands at $\sim 2920$ and $\sim 2850$ $\mathrm{cm}^{-1}$ disappeared completely in the spectrum (single beam mode). The MIRE was mounted in the SBSR flow-through cell immediately after this procedure.

2.3.2. Layer Preparation. Transport of solutions through Teflon tubings was performed by a peristaltic pump (Ismatec SA, Switzerland). First, both cuvettes R and S of Ge MIRE were empty for the measurement of the SBSR reference spectrum of the dry plate. Then, both cuvettes were filled with toluene. The SBSR reference spectrum of toluene was recorded in the stationary state. Before starting the reaction in the $\mathrm{S}$ cuvettes, the $\mathrm{R}$ cuvettes were clamped and remained filled with toluene during the whole experiment. Silanisation in the $\mathrm{S}$ cuvettes was initiated by a fast

(26) Fringeli, U. P.; Baurecht, D.; Günthard Hs. H. Biophysical Infrared Modulation Spectroscopy. In Infrared and Raman Spectroscopy of Biological Materials; Gremlich, H. U., Yan, B., Eds.; Marcel Dekker: New York, 2000; pp $143-192$. exchange of toluene, pumping a $1 \%$ solution of 7-OTCS at a flow rate of $16.5 \mu \mathrm{L} / \mathrm{s}$, leading to a complete exchange of the solution in $\mathrm{S}$ cuvettes within $3 \mathrm{~s}$. After a few seconds, the pumping rate was reduced to $0.83 \mu \mathrm{L} / \mathrm{s}$ and kept constant during the whole experiment acquiring time-resolved single beam spectra over a period of 90 min.

Experiments with CdTe MIRE aimed to give information on the stability of dissolved 7-OTCS by enabling access to the $\mathrm{Si}-\mathrm{Cl}$ stretching vibrations at 587 and $564 \mathrm{~cm}^{-1}$. For that purpose a $2 \%$ solution was inserted into the $\mathrm{S}$ cuvettes and SBSR spectra were acquired. Afterward, the 7-OTCS solution was replaced by toluene and the measurements were repeated.

2.3.3. Oxidation of the Layer. The SBSR cell was connected to a homemade UV chamber containing a small Pen-Ray UV lamp emitting the mercury spectrum with the primary wavelength at 254 $\mathrm{nm}$ inside. Approximately $3 \%$ of the intensity was due to emission at $184 \mathrm{~nm}$, which was responsible for the ozone generation. ${ }^{27}$ Pure oxygen was diluted by argon, using flow rates of $\sim 110 \pm 20 \mathrm{~cm}^{3 /}$ $\min$ for $\mathrm{Ar}$ and $1.4 \pm 0.3 \mathrm{~cm}^{3} / \mathrm{min}$ for $\mathrm{O}_{2}$. Gases were fed into the cell through viton tubes.

Oxidation of the dry layer at $T=15^{\circ} \mathrm{C}$ by gaseous ozone was monitored in situ by ATR spectroscopy. The reference SBSR spectrum of the dried layer was recorded in the stationary state just before oxidation. Time-resolved SBSR spectra were acquired over a time period of $30 \mathrm{~min}$. The acquisition time for one spectrum was typically $220 \mathrm{~s}$ corresponding to $200 \mathrm{scans}$ at a resolution of $4 \mathrm{~cm}^{-1}$. After complete oxidation of the double bond, SBSR spectra of the dry oxidized layer were recorded with parallel and perpendicular polarized light.

2.3.4. Identification of the Expected $\mathrm{COOH}$ Group. Two independent experiments, stationary and ME, were performed in order to prove the existence of $\mathrm{COOH}$ groups produced by oxidation of a terminal ethylenic double bond. For that purpose, the oxidized layer was investigated in aqueous environment at $\mathrm{pH} 4$ and 8 at $T$ $=22{ }^{\circ} \mathrm{C}$.

Stationary and ME experiments were performed on Bruker IFS 25 and IFS 66 spectrometers, respectively. The SBSR cell was connected to two computer-controlled peristaltic pumps working alternatively at flow rates of $1 \mathrm{~mL} / \mathrm{min}$. Both the $\mathrm{R}$ and $\mathrm{S}$ cuvettes were first exposed to a buffer solutions of $\mathrm{pH} 4$ followed by exchange to $\mathrm{pH} 8$ in order to induce periodic protonation and deprotonation of the carboxylic acid groups.

ME spectra were treated as if they were points of a periodic response of a stimulated system. For that purpose, $N=32$ equidistant time-resolved single channel spectra, referred to as sample-point spectra, were recorded within each period of stimulation. Thus, 16 sample-point spectra were associated with the half-period corresponding to $\mathrm{pH} 4$ while the second 16 sample-point spectra were recorded in the half-period where a solution of $\mathrm{pH} 8$ was pumped throughout the cell. These spectra were co-added over six periods. The duration of one cycle was $T=5 \mathrm{~min}$.

2.4. Theoretical Considerations. 2.4.1. Background Compensation. The ATR setup as depicted by Figure 1 enables the performance of a surface chemical reaction in the $\mathrm{S}$ cuvettes while the $\mathrm{R}$ cuvettes serve as reference. Concerning the reaction of 7-OTCS with the Ge surface, two problems have to be overcome for accurate compensation of 7-OTCS solution in contact with the growing layer. The first one results from the fact that the evanescent field penetrates proportionally to its wavelength into the rarer medium. As a consequence the SBSR measurement results in a wavelength-dependent overcompensation of the background solution due to its displacement by the growing layer. One has to take into account that the influence of the layer thickness is more prominent at high wavenumbers (small wavelengths). Therefore, basic ATR theory has always to be applied for compensating the background in contact with a surface layer.

In the case of organic materials, it was already shown by the independent inventors of ATR spectroscopy, N. J. Harrick ${ }^{28}$ and J.

(27) Norrod, K. L.; Rowlen, K. L. J. Am. Chem. Soc. 1998, 120, 2656-2657.

(28) Harrick, N. J. and du Pré, F. K. Appl. Opt. 1966, 5, 1739-1743. 
Fahrenfort, ${ }^{29}$ that the absorption index, $k$, in the complex refractive index, $\hat{n}$, eq 4 , is generally $<0.1$.

$$
\hat{n}=n+i k
$$

Under this condition, the influence on Fresnel's reflection coefficients is rather low, justifying quantitative analysis using relative electric field strength in the rarer medium calculated for a nonabsorbing medium, i.e., $k=0$. On the basis of this approximation, the concept of effective thickness, $d_{\mathrm{e}}$, was developed. A sample of hypothetical thickness $d_{\mathrm{e}}$ would result in the same absorbance in a transmission experiment as in the real internal reflection experiment. For bulk isotropic rarer material of refractive index, $n_{3}$, one obtains

$$
d_{\mathrm{e}, \mathrm{pp} / \mathrm{vp}}^{\mathrm{b}, \mathrm{iso}}=\frac{n_{3}}{n_{1} \cos \theta} \frac{d_{\mathrm{p}}}{2} E_{\mathrm{b}, \mathrm{pp} / \mathrm{vp}}^{r 2}
$$

where $d_{\mathrm{p}}$ denotes the wavelength-dependent penetration depth, as indicated by eq 6 , and $\mathrm{b}$ stands for bulk. $n_{1}, n_{2}$ and $n_{3}$, are the refractive indices for MIRE, thin layer, and bulk environment, respectively.

$$
d_{\mathrm{p}}=\frac{\lambda}{2 \pi n_{1}\left(\sin ^{2} \theta-\left(\frac{n_{3}}{n_{1}}\right)^{2}\right)^{1 / 2}}
$$

$E_{\mathrm{b}, \mathrm{pp} / \mathrm{vp}}^{r}$ denotes the relative electric field strength in the bulk (b) rarer medium for parallel (pp) and perpendicular (vp) polarized incident light, respectively. ${ }^{28}$ Since $E_{\mathrm{pp} / \mathrm{vp}}^{r}$ declines exponentially with the distance, $z$, from the interface,

$$
E_{\mathrm{pp} / \mathrm{vp}}^{r}=E_{\mathrm{pp} / \mathrm{vp}, 0}^{r} \mathrm{e}^{-z / d_{\mathrm{p}}}
$$

eq 5 converts to eq 8 in case of a very thin $\left(d \ll d_{\mathrm{p}}\right)$ isotropic surface layer (th) of thickness $d$ and refractive index $n_{2}$,

$$
d_{\mathrm{e}, \mathrm{pp} / \mathrm{vp}}^{\mathrm{th}, \mathrm{si}}=\frac{n_{2}}{n_{1} \cos \theta} d E_{\mathrm{th}, \mathrm{pp} / \mathrm{vp}}^{r 2}
$$

For adequate background compensation in the sample channel S, it follows from eqs 5 and 8 that the absorbance spectrum of the pure solution measured in the $\mathrm{R}$ channel has to be scaled by the factor $\kappa$ and then added to the experimentally obtained SBSR absorbance spectrum, thus removing the wavelength-dependent overcompensation.

$$
\kappa=\frac{d_{\mathrm{e}, \mathrm{pp} / \mathrm{vp}}^{\mathrm{th}, \mathrm{iso}}}{d_{\mathrm{e}, \mathrm{pp} / \mathrm{vp}}^{\text {biso }}}=\frac{2 d}{d_{\mathrm{p}}}=4 \pi\left(n_{1}{ }^{2} \sin ^{2} \theta-n_{3}{ }^{2}\right)^{1 / 2} \tilde{\nu} d
$$

It should be noted that the layer displaced solution has the same refractive index as the bulk. Therefore, one has to set $n_{2}=n_{3}$ in eq 8.

The scaling factor, $\kappa$, is independent of the polarization. For a layer thickness of $d=13 \AA, n_{1}=4.0(\mathrm{Ge}), n_{3}=1.49$ (toluene, bulk), $\theta=45^{\circ}$ (angle of incidence), one obtains for $\kappa$ as depending on the wavenumber, $\tilde{v}$, the following linear graph (Figure 2).

The second problem in background compensation results from the fact that, in the case of a chlorosilane compound, it is principally not possible to measure an ATR spectrum with a Ge MIRE unless chemical reaction immediately starts at the surface, but this problem also could be overcome by theoretical means. Details of this procedure are reported in ref 24 .

For the sake of shortness, only the principles of this method will be described here. The final time-resolved absorbance spectrum immediately before termination of the reaction between Ge and 7-OTCS was taken as base. Toluene overcompensation in this SBSR spectrum occurred due to its displacement by the attached layer as

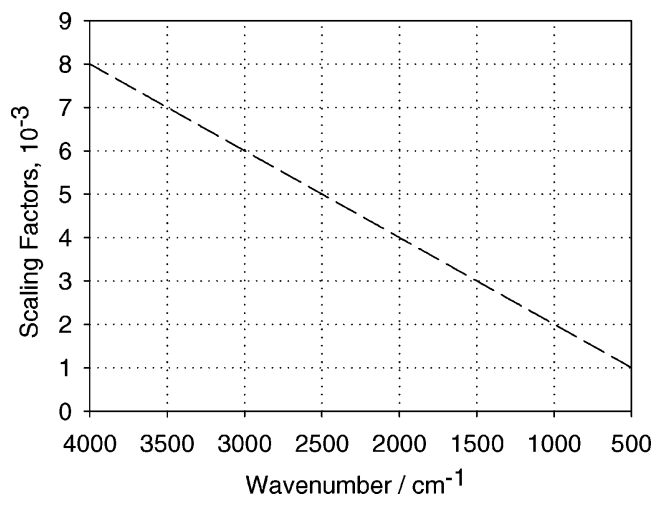

Figure 2. Dependence of the scaling factor, $\kappa$, on the wavenumber, $\tilde{v}$. The solvent overcompensation in presence of a thin layer $(d=$ $1.3 \mathrm{~nm}$ ) corresponds to the product of $\kappa$ with the absorbance spectrum of pure solvent in contact with the MIRE. For details see ref 24.

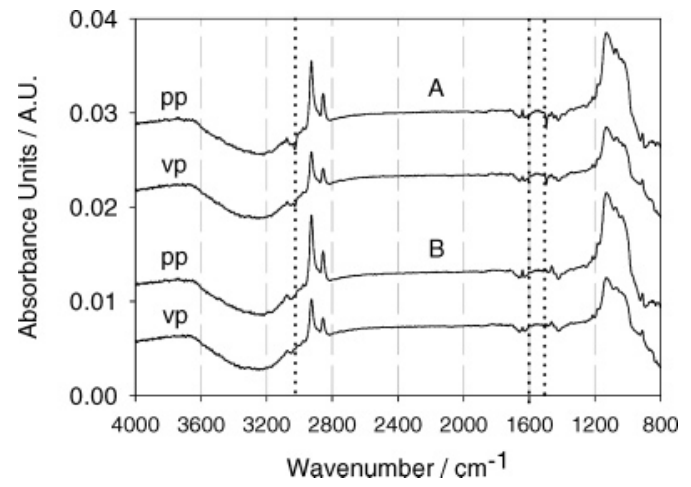

Figure 3. SBSR spectra of a silane layer bound to the Ge MIRE in the $\mathrm{S}$ cuvettes, (A) without and (B) with toluene compensation. The broad negative band near $3300 \mathrm{~cm}^{-1}$ and the negative band at $1640 \mathrm{~cm}^{-1}$ are expected to result from a thin water layer removed in the $\mathrm{S}$ cuvettes due to chemical reaction with 7-OTCS; however, in the $\mathrm{R}$ cuvettes the water layer was too tightly attached at the $\mathrm{Ge}$ surface and could not be removed by dry toluene. Typical absorption bands of 7-OTCS may be assigned: $v_{\text {as }}\left(\mathrm{CH}_{2}\right)$ and $v_{\mathrm{s}}\left(\mathrm{CH}_{2}\right)$ at 2927 and $2855 \mathrm{~cm}^{-1}, \delta\left(\mathrm{CH}_{2}\right)$ at $1463 \mathrm{~cm}^{-1}, v_{\text {as }}\left(\mathrm{CH}=\mathrm{CH}_{2}\right)$ at $3077 \mathrm{~cm}^{-1}$, $v(\mathrm{C}=\mathrm{C})$ at $1641 \mathrm{~cm}^{-1}$ and $\gamma_{\mathrm{op}}\left(\mathrm{CH}=\mathrm{CH}_{2}\right)$ at $911 \mathrm{~cm}^{-1}$. Finally, the bands featuring connection of 7-OTCS to the Ge surface or 7-OTCS polymerization form a broad low structured band between 1250 and $975 \mathrm{~cm}^{-1}$ reflecting vibration such as $v(\mathrm{Ge}-\mathrm{O}-\mathrm{Si}), v(\mathrm{Si}-\mathrm{O}-\mathrm{Si})$, and $v(\mathrm{Si}-\mathrm{OH})$. All spectra were measured with parallel $(\mathrm{pp})$ and perpendicular (vp) polarized light. $T=15^{\circ} \mathrm{C}$; angle of incidence $\theta=45^{\circ}$; number of active internal reflections $N=19.4$.

well as by its dilution by 7-OTCS. Reaction of 7-OTCS was stopped by replacing the silane solution by pure toluene and measuring again the corresponding absorbance spectrum. In this case overcompensation resulted only from solvent displacement by the layer (Figure $3 \mathrm{~A})$. Background compensation according to eq 8 and Figure 2 resulted in the spectra of bound silane (Figure 3B). These spectra were now subtracted from the corresponding absorbance spectra resulting from the final silane layer still in contact with 7-OTCS solution, resulting in the absorbance spectra of 7-OTCS solution in the range from distance $d$ (layer thickness) to infinity. The experimentally not accessible reference spectra of 7-OTCS in toluene could now be calculated by a scaling procedure as described before. All details are reported in ref 24.

2.4.2. Orientation Measurements. Orientation measurements require independent spectra of a sample measured with parallel and perpendicular polarized incident light. The basic quantity for data analysis is the so-called dichroic ratio $R(\tilde{v})=\int A_{\mathrm{pp}}(\tilde{v}) \mathrm{d} \tilde{v} / \int A_{\mathrm{vp}}$ $(\tilde{v}) \mathrm{d} \tilde{v}$, where $\int A_{\mathrm{pp}}(\tilde{v}) \mathrm{d} \tilde{v}$ and $\int A_{\mathrm{vp}}(\tilde{v}) \mathrm{d} \tilde{v}$ denote the integrated absorbances of a given absorption band measured with parallel (pp) and perpendicular (vp) polarized incident light, respectively. Peak absorbances may also be used instead of integrated absorbances. Assuming a liquid crystalline ultrastructure (LCU) of the sample 
with uniaxial orientation along the normal to the MIRE ( $z$ axis), one obtains for the dichroic ratio the following expression: ${ }^{30}$

$$
R=\frac{\int A_{\mathrm{pp}}(\tilde{v}) \mathrm{d} \tilde{v}}{\int A_{\mathrm{vp}}(\tilde{v}) \mathrm{d} \tilde{v}}=\frac{E_{x}^{2}}{E_{y}^{2}}+2 \frac{E_{z}^{2} \overline{\left\langle\cos ^{2} \Theta\right\rangle}}{E_{y}{ }^{2}\left(1-\overline{\left\langle\cos ^{2} \Theta\right\rangle}\right)}
$$

$\left\langle\overline{\left.\cos ^{2} \Theta\right\rangle}\right.$ denotes the average over the mean squares of the cosines of the angles between the transition moments of a given vibration and the $z$ axis of the laboratory coordinate system which is fixed to the MIRE. The $x y$ plane is parallel to the surface of the MIRE, with the $x$ axis directing along the light propagation. For details, the reader is referred to ref 30 .

2.4.3. Determination of Surface Concentration. The volume concentration, $c$, and the surface concentration, $\Gamma$, are related to each other via the thickness of the sample, $d$. By introducing Lambert-Beer's law, one obtains

$$
c=\frac{\Gamma}{d}=\frac{\int A_{\mathrm{pp} / \mathrm{vp}}(\tilde{v}) \mathrm{d} \tilde{v}}{N v d_{\mathrm{e}, \mathrm{pp} / \mathrm{vp}}^{\mathrm{th}} \int \epsilon(\tilde{v}) \mathrm{d} \tilde{v}}
$$

$N$ and $v$ are the mean number of the active internal reflections and the number of equal functional groups per molecule (e.g., $N=$ 19.4 , and $v=6 \mathrm{CH}_{2}$ groups in 7-OTCS). $d_{\mathrm{e}, \mathrm{pp} / \mathrm{vp}}^{\text {th }}$ is the effective thickness of the layer. It depends on the polarization of incident light and on the structure of the layer..$^{30} \int \epsilon(\tilde{v}) \mathrm{d} \tilde{v}$ denotes the decadic integrated molar absorption coefficient of the band. The surface concentration, $\Gamma$, may then be expressed as depending on the experimentally determined dichroic ratio, $R$, and the relative electric field strengths resulting from Fresnel's equations

$$
\Gamma=\frac{d \int A_{\mathrm{pp} / \mathrm{vp}}(\tilde{v}) \mathrm{d} \tilde{v}}{3 N \nu d_{\mathrm{e}, \mathrm{pp} / \mathrm{vp}}^{\mathrm{th}, \mathrm{iso}} \int \epsilon(\tilde{v}) \mathrm{d} \tilde{v}}\left[2-\frac{E_{x}^{r 2}}{E_{z}^{r 2}}+R \frac{E_{y}^{r 2}}{E_{z}^{r 2}}\right]
$$

The surface concentration, $\Gamma$, as given by eq 12 may be understood as the projection of the molecules in the volume defined by unit area and height $d$ (real sample thickness). Accordingly to eq 8, eq 12 converts into eq 13 in the case of a thin layer.

$$
\Gamma=\frac{\int A_{\mathrm{pp} / \mathrm{vp}}(\tilde{v}) \mathrm{d} \tilde{v}}{3 N \nu d_{\mathrm{e}, \mathrm{pp} / \mathrm{vp}}^{\mathrm{rel}, \mathrm{iso}} \int \epsilon(\tilde{v}) \mathrm{d} \tilde{v}}\left[2-\frac{E_{x}^{r 2}}{E_{z}^{r 2}}+R \frac{E_{y}^{r 2}}{E_{z}^{r 2}}\right]
$$

As a consequence, surface concentration of a thin layer $\left(d \ll d_{\mathrm{p}}\right)$ featuring uniaxial orientation along the $z$ axis can be determined without knowing the real thickness, $d$, and its real structure. Structural information of the layer is contained in the experimentally determined dichroic ratio, $R$.

\section{Results}

3.1. Determination of Integrated Molar Absorption Coefficients of Characteristic Bands of 7-OTCS. Integrated molar absorption coefficients of 7-OTCS in toluene were determined by transmission measurements using a $\mathrm{CaF}_{2}$ cell with $l=10 \mu \mathrm{m}$ path length. Four concentrations of 7-OTCS in toluene (1\%, $2 \%$, $4 \%, 7.75 \%(\mathrm{v} / \mathrm{v}))$ were used (see Table 1). Integrated molar

(30) Fringeli, U. P.; Baurecht, D.; Bürgi, T.; Siam, M.; Reiter, G.; Schwarzott, M.; Brüesch, P. ATR Spectroscopy of Thin Films. In Handbook of Thin Films Materials; Nalwa, H. S., Ed.; Academic Press: New York, 2002; Vol. 2, Chapter 4, pp 191-229.
Table 1. Integrated Molar Absorption Coefficients of 7-OTCS in Toluene Determined by Transmission Measurements

\begin{tabular}{lll}
\hline \multicolumn{1}{c}{$c_{\mathrm{CH} 2, \mathrm{OTCS}} / \mathrm{mol} / \mathrm{L}$} & \multicolumn{1}{c}{$v_{\mathrm{s}}\left(\mathrm{CH}_{2}\right)$} & \multicolumn{1}{c}{$v_{\mathrm{as}}\left(\mathrm{CH}_{2}\right)$} \\
\hline & $\int A(\tilde{v}) d \tilde{v} / \mathrm{cm}^{-1}$ & $\int A(\tilde{v}) d \tilde{v} / \mathrm{cm}^{-1}$ \\
& $2873-2823 \mathrm{~cm}^{-1}$ & $2968-2898 \mathrm{~cm}^{-1}$ \\
0.261 & 0.0698 & 0.2756 \\
0.523 & 0.1461 & 0.5565 \\
1.05 & 0.3061 & 1.1253 \\
2.03 & 0.5901 & 2.1982 \\
$\int \epsilon(\tilde{v}) d \tilde{v} / 10^{5} \mathrm{~cm} / \mathrm{mol}$ & $2.95 \pm 0.14$ & $10.9 \pm 0.5$
\end{tabular}

Table 2. Magnitudes and Uncertainties of Input Parameter

\begin{tabular}{ll}
\hline \multicolumn{1}{c}{ parameter, symbol } & \multicolumn{1}{c}{ magnitude } \\
\hline angle of incidence, $\theta /$ deg & $45.0 \pm 1.5$ \\
refractive index of germanium MIRE, $n_{1}$ & $4.0 \pm 0.0$ \\
refractive index of an adsorbed/bound layer, $n_{2}$ & $1.45 \pm 0.05$ \\
refractive index of toluene environment, $n_{3}$ & $1.49 \pm 0.05$ \\
integrated molar absorption coefficient of $\nu_{\mathrm{s}}\left(\mathrm{CH}_{2}\right)$, & $2.95 \pm 0.14$ \\
$\int \epsilon(\tilde{v}) \mathrm{d} \tilde{v} / 10^{5} \mathrm{~cm} / \mathrm{mol}$ & \\
integrated molar absorption coefficient of $\nu_{\mathrm{as}}\left(\mathrm{CH}_{2}\right)$, & $10.9 \pm 0.5$ \\
$\int \epsilon(\tilde{v}) \mathrm{d} \tilde{v} / 10^{5} \mathrm{~cm} /$ mol & \\
number of equal functional groups per molecule, $v$ & $6 \pm 0$ \\
number of active internal reflections, $N$ & $19.4 \pm 1$
\end{tabular}

absorption coefficients were determined according to LambertBeer's law:

$$
\int \epsilon(\tilde{v}) \mathrm{d} \tilde{v}=\frac{\int A(\tilde{v}) \mathrm{d} \tilde{v}}{c_{\mathrm{CH}_{2}, \mathrm{OTCS}} l}
$$

The results are summarized in Table 1 . In the case of $\mathrm{CH}_{2}$ vibrations, the integrated absorbancies were divided by $N=6$, the number of $\mathrm{CH}_{2}$ groups per molecule.

3.2. Silanization of a Ge MIRE. 3.2.1. Final State of Silanization. Figure 3 shows SBSR spectra of the final state of a typical silanization experiment. The silane layer was bound to the Ge MIRE in the S channel, (A) without and (B) with background compensation according to eq 9 . We suggest that the broad negative bands near 3300 and $1640 \mathrm{~cm}^{-1}$ result from a compact water monolayer ${ }^{31}$ with a thickness of about $3 \AA$, which was removed by the reaction with 7-OTCS, however, remained unaffected by dry toluene in the $\mathrm{R}$ channel. No explanation for the third weak negative and close to $1420 \mathrm{~cm}^{-1}$ can be given at the moment.

Some further assignments should be mentioned. $v_{\mathrm{as}}\left(\mathrm{CH}_{2}\right)$ and $v_{\mathrm{s}}\left(\mathrm{CH}_{2}\right)$ at 2927 and $2855 \mathrm{~cm}^{-1}, \delta\left(\mathrm{CH}_{2}\right)$ at $1463 \mathrm{~cm}^{-1}, v_{\mathrm{as}^{-}}$ $\left(\mathrm{CH}=\mathrm{CH}_{2}\right)$ at $3077 \mathrm{~cm}^{-1}, v(\mathrm{C}=\mathrm{C})$ at $1641 \mathrm{~cm}^{-1}$, and $\gamma_{\mathrm{op}}(\mathrm{CH}=$ $\left.\mathrm{CH}_{2}\right)$ at $911 \mathrm{~cm}^{-1}$. The reactive side of 7-OTCS is characterized by $v_{\mathrm{s}}\left(\mathrm{SiCl}_{3}\right)$ and $\nu_{\mathrm{as}}\left(\mathrm{SiCl}_{3}\right)$ at 564 and $587 \mathrm{~cm}^{-1}$. Finally, the bands featuring chemical connection of 7-OTCS molecules to the Ge surface or 7-OTCS polymerization exhibit a broad low structured shape between $1250-975 \mathrm{~cm}^{-1}$ reflecting vibrations such as $v(\mathrm{Ge}-\mathrm{O}-\mathrm{Si}), v(\mathrm{Si}-\mathrm{O}-\mathrm{Si})$, and $v(\mathrm{Si}-\mathrm{OH})^{32}$.

The relevant parameters used for quantitative analysis are summarized in Table 2. The surface concentration of the final state of the silane layer in the $\mathrm{S}$ cuvettes was calculated by means of eq 13 using integrated absorbances of symmetric and asymmetric $\mathrm{CH}_{2}$ stretching vibrations resulting in $\Gamma_{\exp }=(6.33$ $\pm 0.70) \times 10^{-10} \mathrm{~mol} / \mathrm{cm}^{2}$. From this quantity, one may estimate the mean layer thickness $d$, based on its density $\rho \approx 0.90 \pm 0.10$ $\mathrm{g} / \mathrm{cm}^{33}$ and molecular mass $M_{\mathrm{r}}=187.15 \mathrm{~g} / \mathrm{mol}$ to be $d \approx$ $\left(\Gamma_{\exp } M_{\mathrm{r}}\right) / \rho=1.3 \pm 0.2 \mathrm{~nm}$. It should be noted that $\mathrm{Si}-\mathrm{Cl}$ was

(31) Brzoska, J. B.; Azouz, I. B.; Rondelez, F. Langmuir 1994, 10, 11, 43674373.

(32) Matinlinna, J. P.; Areva S.; Lassila, L. V. J.; Vallittu, P. K. Surf. Interface Anal. 2004, 36, 1314-1322. 
Table 3. Summary of Quantitative Data on Surface Concentration and Orientation of Silane Layer Based on the Evaluation of $\mathrm{CH}_{2}$ Symmetric and Asymmetric Stretching Vibrations

\begin{tabular}{lll}
\hline & \multicolumn{1}{c}{$v_{\mathrm{s}}\left(\mathrm{CH}_{2}\right)$} & \multicolumn{1}{c}{$v_{\mathrm{as}}\left(\mathrm{CH}_{2}\right)$} \\
\hline integration limits $/ \mathrm{cm}^{-1}$ & $2873-2823$ & $2968-2898$ \\
$\int A_{\mathrm{pp}}(\tilde{v}) \mathrm{d} \tilde{v}$ & $0.044 \pm 0.003$ & $0.192 \pm 0.001$ \\
$\int A_{\mathrm{vp}}(\tilde{v}) \mathrm{d} \tilde{v}$ & $0.028 \pm 0.002$ & $0.117 \pm 0.006$ \\
$R_{\exp }$ & $1.55 \pm 0.15$ & $1.64 \pm 0.08$ \\
$\Gamma_{\exp } / 10^{-10} \mathrm{~mol} / \mathrm{cm}^{2} a$ & $5.92 \pm 0.69$ & $6.75 \pm 0.69$ \\
$d / \mathrm{nm}$ & 1.23 & 1.40 \\
$\left\langle\cos ^{2} \Theta\right\rangle^{b}$ & 0.21 & 0.24 \\
$\Theta / \mathrm{deg}^{c}$ & $62.4 \pm 0.5$ & $60.9 \pm 0.4$ \\
$S_{\text {seg }}{ }^{c}$ & $-0.17 \pm 0.01$ & $-0.145 \pm 0.009$ \\
$S_{\text {mol }}{ }^{c}$ & $0.71 \pm 0.37$ & $0.57 \pm 0.30$
\end{tabular}

${ }^{a}$ Surface concentration calculated from eq 13. ${ }^{b}$ Mean direction of $\mathrm{CH}_{2}$ transition moments, calculated from eq $10 .{ }^{c}$ Segmental and molecular order parameter of hydrocarbon chain, see ref 30.

replaced by $\mathrm{Si}-\mathrm{O}$ when calculating $M_{\mathrm{r}}$ since no $\mathrm{Si}-\mathrm{Cl}$ vibrations were detectable in the final layer, as documented by measurements with a CdTe MIRE, see later.

For the estimation of upper and lower limits for the area per 7-OTCS molecule after immobilization on the Ge plate, two boundary states were considered from molecular modeling calculation (HyperChem (TM), Hypercube, Inc., Gainesville, FL). Aligning the hydrocarbon chain vertically would result in the minimum area of $A_{\min }=34 \AA^{2} /$ molecule which corresponds to a surface concentration of $\Gamma_{\max }=4.88 \times 10^{-10} \mathrm{~mol} / \mathrm{cm}^{2}$. If the molecules lie on the Ge plate, they occupy an area of $A_{\max }$ $=69 \AA^{2} /$ molecule, which corresponds to a surface concentration of $\Gamma_{\min }=2.41 \times 10^{-10} \mathrm{~mol} / \mathrm{cm}^{2}$. The experimentally determined mean surface concentration of $\Gamma_{\exp }=6.33 \times 10^{-10} \mathrm{~mol} / \mathrm{cm}^{2}$ is about $30 \%$ above the most densely packed monolayer coverage and indicates that most probably polymerization has occurred as a parallel reaction to covalent binding to the Ge surface.

The mean direction of the transition moments of symmetric and asymmetric $\mathrm{CH}_{2}$ stretching vibrations, $v_{\mathrm{s}}\left(\mathrm{CH}_{2}\right)$ and $v_{\mathrm{as}}\left(\mathrm{CH}_{2}\right)$, with respect to the $z$ axis may be estimated from the corresponding experimentally determined dichroic ratios, $R$, according to eq 10. The relevant data are summarized in Table 3. Averaging the dichroic ratios of both vibrations results in $R_{\mathrm{CH} 2 \text {,mean }}=1.60 \pm$ 0.09 , which results in a mean angle between the corresponding transitions moments and the $z$ axis of $\Theta=61.6^{\circ} \pm 0.3^{\circ}$. Since these transition moments are normal to the local molecular axis, ${ }^{26}$ one may estimate the mean direction of the hydrocarbon chains of the silane assembly to be inclined by $\delta=28.4^{\circ} \pm 0.3^{\circ}$ against the normal to the MIRE ( $z$ axis). It should be noted that an isotropic thin layer would exhibit a dichroic ratio of $R_{\text {iso }}=2.13$ \pm 0.27 , which is significantly different from the experimental value. This finding is supported by the calculation of the mean segmental order parameter of the hydrocarbon chain $S_{\mathrm{seg}}=-0.16$ \pm 0.01 and the corresponding molecular order parameter $S_{\mathrm{mol}}=$ $0.64 \pm 0.34$. For perfect alignment of the hydrocarbon chain along the normal to the plate, one would expect $S_{\mathrm{seg}, \mathrm{z}}=-0.50$ and $S_{\mathrm{mol}, \mathrm{z}}=1.00$, see Table 3 and ref 30 .

3.2.2. Kinetics of Layer Formation. Figure 4 shows some selected background compensated time-resolved spectra measured during layer growth. The spectra were measured with parallel polarized incident light after $6,18,30,42$, and 54 min of exposition to a $1 \%$ solution of 7 -OTCS in toluene. It should be noted that at the very beginning of the reaction a broad band reflecting the formation of $\mathrm{Ge}-\mathrm{O}-\mathrm{Si}(v(\mathrm{Ge}-\mathrm{O}-\mathrm{Si}))$ appeared at $1000 \mathrm{~cm}^{-1}$.

(33) Rühe, J.; Novotny, V. J.; Kanazawa, K. K.; Clarke T.; Street, G. B. Langmuir 1993, 9, 2383-2388.

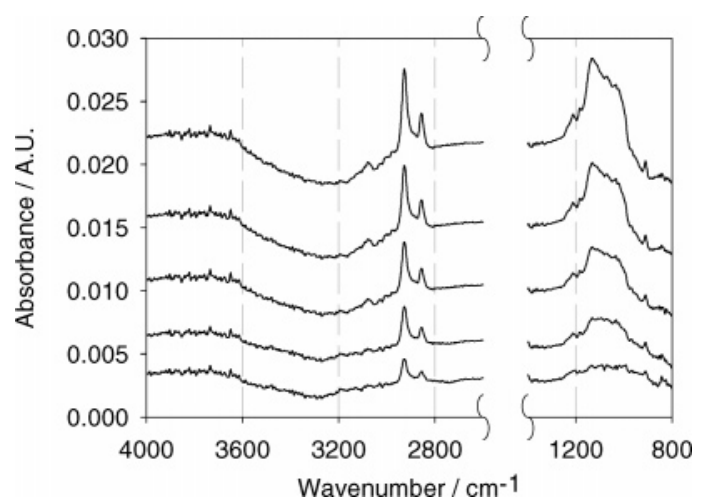

Figure 4. Selected background compensated time-resolved FTIR ATR spectra of the silane layer in contact with 1\% 7-OTCS solution showing growth of the layer on a Ge MIRE after 6, 18, 30, 42, and $54 \mathrm{~min}$ from the beginning of silanization reaction. Reference: toluene. Compensation of 7-OTCS solution was performed according to the procedure described in Section 2.4.1. For band assignment see Figure 3 and text. Note that reaction with the Ge MIRE is clearly documented by the growing of the $1250-975 \mathrm{~cm}^{-1}$ band complex which is assigned predominantly $\mathrm{Ge}-\mathrm{O}-\mathrm{Si}$ vibrations. Partial overlapping by $\mathrm{Si}-\mathrm{O}-\mathrm{Si}$ vibrations are expected due to polymerization. $T=15^{\circ} \mathrm{C}$; angle of incident light $\theta=45^{\circ}$; number of active internal reflections $N=19.4$.

As concluded from Figure 4 this band dominates the spectral region within the first $30 \mathrm{~min}$. Then, a continuous shift of this band to higher wavenumbers is observed, paralleled by a slower rate of layer growth. This process is assigned to polymerization, i.e., to the formation of $\mathrm{Si}-\mathrm{O}-\mathrm{Si}$ groups by the reaction of $\mathrm{Si}-\mathrm{Cl}_{3}$ of silane in solution with $\mathrm{Si}-\mathrm{OH}$ groups of bound silane. Analysis of the kinetics of a typical experiment will be given in ref 24.

Typical absorption bands of 7-OTCS are clearly resolved, such as the two most significant $\mathrm{CH}_{2}$ stretching vibrations $v_{\mathrm{s}^{-}}$ $\left(\mathrm{CH}_{2}\right)$ and $v_{\text {as }}\left(\mathrm{CH}_{2}\right)$ and $v_{\text {as }}\left(\mathrm{CH}=\mathrm{CH}_{2}\right)$ at $3077 \mathrm{~cm}^{-1}$, as well as the corresponding out-of-plane deformation $\gamma_{\mathrm{op}}\left(\mathrm{CH}=\mathrm{CH}_{2}\right)$ at $911 \mathrm{~cm}^{-1}$. The broad band complex in the $1100 \mathrm{~cm}^{-1}$ region indicates the formation of $\mathrm{Ge}-\mathrm{O}-\mathrm{Si}$ bonds at the MIRE surface and probably only in the later phase also polymerization of 7-OTCS leading to $\mathrm{Si}-\mathrm{O}-\mathrm{Si}$ bonds. Layer formation is paralleled by the interesting kinetic behavior of the broad negative band centered at $3300 \mathrm{~cm}^{-1}$. This band is correlated with a weak also negative band at $1640 \mathrm{~cm}^{-1}$ (see Figure 3), both originating most probably from small amounts of water displaced in the $S$ cuvettes or introduced in the R cuvettes. We relate these bands tentatively to a thin water layer ${ }^{31}$ at the Ge MIRE which remained stable, even in contact with dry toluene. We suggest that $\mathrm{H}_{2} \mathrm{O}$ is removed by the chemical reaction with $\mathrm{Si}-\mathrm{Cl}_{3}$ of 7-OTCS leading to $\mathrm{HCl}$ and $\mathrm{Si}-\mathrm{OH}$ thus enabling polymerization of 7-OTCS.

CdTe was used as MIRE in order to have access to $v_{\mathrm{s}}\left(\mathrm{SiCl}_{3}\right)$ and $v_{\text {as }}\left(\mathrm{SiCl}_{3}\right)$ at 564 and $587 \mathrm{~cm}^{-1}$. Moreover, since dry CdTe exhibits a hydrophobic surface, one could expect that no tightly bound water layers would be present, as in the case of Ge. Figure $5 \mathrm{~A}$ shows the state of $2 \%$ OTCS solution in toluene after $\sim 9 \mathrm{~h}$ of contact with the CdTe MIRE. The presence of $-\mathrm{SiCl}_{3}$ moieties is clearly proved by the corresponding $\mathrm{Si}-\mathrm{Cl}$ stretching vibrations. However, unexpectedly, 7-OTCS adsorbed also irreversibly to $\mathrm{CdTe}$, even without $-\mathrm{OH}$ groups at the surface. This is revealed upon exchanging 7-OTCS solution by pure toluene, Figure 5B. There remained a tightly bound layer on the CdTe surface, lacking of any $\mathrm{Si}-\mathrm{Cl}$ bonds. We suggest that the broad band in the 3400 $\mathrm{cm}^{-1}$ region results predominantly from $v(\mathrm{OH})$ vibrations of $\mathrm{Si}-\mathrm{OH}$, enabling polymerization at the interface. Since this process turned out to be very slow $(9 \mathrm{~h})$, we expect that very 


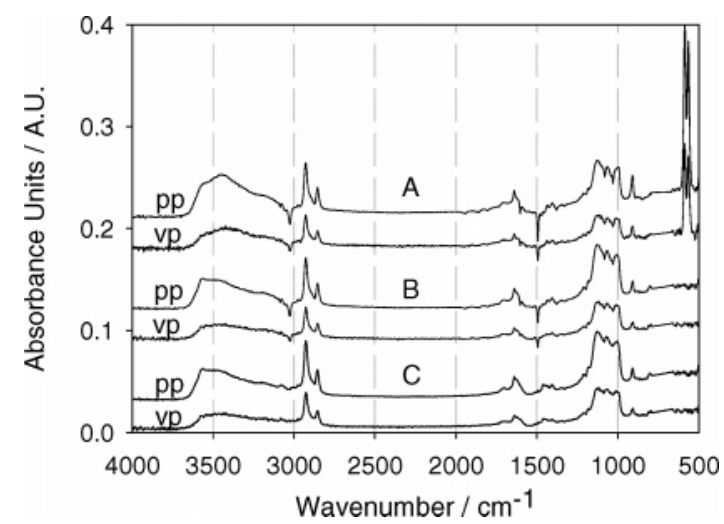

Figure 5. Parallel (pp) and perpendicular (vp) polarized SBSR spectra of the physically bound layer to the CdTe MIRE. (A) Adsorbed silane layer in contact with $2 \%$ 7-OTCS solution (v/v) where $\mathrm{SiCl}_{3}$ stretching vibrations placed at 587 and $565 \mathrm{~cm}^{-1}$ dominate in the spectra. (B and C) Adsorbed silane layer in contact with toluene have lost all $\mathrm{SiCl}_{3}$ stretching vibrations due to complete polymerization to either a $\mathrm{Si}-\mathrm{O}-\mathrm{Si}\left(1250-975 \mathrm{~cm}^{-1}\right)$ in oligomers or $\mathrm{Si}-$ $\mathrm{OH}$ bands $\left(3600-3100 \mathrm{~cm}^{-1}\right)$ without and with toluene compensation, respectively. $T=15^{\circ}$; angle of incidence $\theta=45^{\circ}$; refractive index $n_{1}=2.67$; number of active internal reflections $N=12.7$.

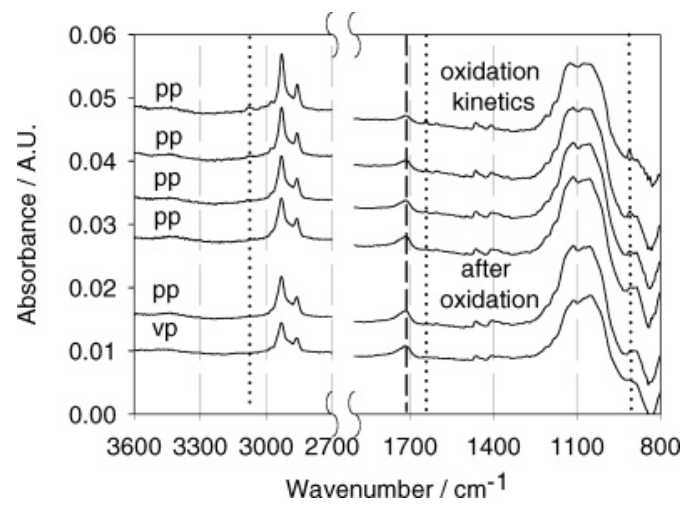

Figure 6. Oxidation of the dry silane layer by ozone produced by means of UV irradiation of an $\mathrm{Ar} / \mathrm{O}_{2}$ mixture. On top there are time-resolved SBSR spectra recorded after 2, 14, 21, and $32 \mathrm{~min}$ of exposure to ozone. The last two spectra on the bottom are SBSR spectra measured after complete oxidation of the double bond. A new band appeared at $1718 \mathrm{~cm}^{-1}$ featuring the reaction product $-\mathrm{COOH}$. However, it should be noted that $v_{\mathrm{s}}\left(\mathrm{CH}_{2}\right)$ decreased also by $46 \%$, reflecting that even parts of the saturated hydrocarbon chains have been oxidized during ozone exposure. $T=15^{\circ} \mathrm{C}$; angle of incident light $\theta=45^{\circ}$; number of active internal reflections $N$ $=19.4$.

small quantities of $\mathrm{H}_{2} \mathrm{O}$ still in dried toluene has been responsible for $\mathrm{Si}-\mathrm{Cl}$ hydrolization. Figure $5 \mathrm{C}$ presents the physisorbed polymerized silane layer with complete background compensation. From the latter we estimate a mean thickness of $11 \mathrm{~nm}$.

3.2.3. Oxidation of Terminal Double Bond of the Silane Layer. The aim of this step is to produce immobilized 7-carboxylsilane, i.e., a layer of carboxylic acid groups facing the bulk. These groups are then intended to be activated for further chemical immobilization steps. Double bond oxidation was performed by exposing the dry silane layer to Ar diluted ozone $\left(\mathrm{O}_{3}\right)$. For details see Section 2.3.3. The results are shown in Figure 6. On top there are four selected spectra measured 2, 14, 21, and 32 min after the onset of the ozone flow. The disappearance of typical absorption bands of the double bond is visible at 3077, 1641, and $911 \mathrm{~cm}^{-1}$. This effect is paralleled by the appearance of a new band at $1718 \mathrm{~cm}^{-1}$ which is tentatively assigned to $v(\mathrm{C}=\mathrm{O})$ of $-\mathrm{COOH}$ or to an aldehyde $-\mathrm{CHO}$. Spectra of the dry layer after oxidation were measured with $\mathrm{pp}$ - and vp-polarized incident light and are shown on the bottom of Figure 6. It should be noted that oxidation did not stop completely after removing the endstanding double bond; about $46 \%$ of the saturated hydrocarbon chains have also been removed under the applied conditions.

We suppose that the following mechanism of oxidation has taken place. ${ }^{34,35}$ The ozone initially attacks double bond forming the primary ozonide. The primary ozonide decomposes releasing formaldehyde to the gas-phase leaving the Criegee intermediate on the surface. The Criege intermediate then rearranges on the surface and decomposes yielding carbon dioxide in the gas phase and a surface bound alkyl chain with carboxylic ends. However, it should be mentioned that upon oxidation the intensity of the $\mathrm{CH}_{2}$ stretching bands were significantly reduced. The explanation could be an arbitrary continued oxidation of $\mathrm{CH}_{2}$ groups or cutting the whole hydrocarbon chain from the $\mathrm{Si}-\mathrm{O}-\mathrm{Ge}$ interface. After extended exposure of the layer to ozone, the broad band at 1000 $\mathrm{cm}^{-1}$ which is assigned to $v(\mathrm{Ge}-\mathrm{O}-\mathrm{Si})$ remained unaffected while $v\left(\mathrm{CH}_{2}\right)$ vanished completely (spectra not shown).

Obviously, the terminal ethylenic double bond is very sensitive to oxidation, since in some experiments partial oxidation could already be observed during layer formation still in toluene environment. This unexpected phenomenon probably was facilitated by small particles of molecular sieve which remained in toluene after the drying process. An atomic force microscopic investigation revealed particles in the sub-micrometer range sitting homogeneously distributed in the silane layer. ${ }^{36,37}$

3.2.4. Proof of the Existence of an End-Standing $-\mathrm{COOH}$ Group. The band at $1718 \mathrm{~cm}^{-1}$ appearing during the oxidation process is a strong evidence for the aimed product. Validation was performed by exposing the oxidized layer to aqueous solutions at $\mathrm{pH} 4$ and 8 , respectively. If the end-standing group is really $-\mathrm{COOH}$, one would expect deprotonation at $\mathrm{pH} 8$ leading to two characteristic bands near 1550 and $1400 \mathrm{~cm}^{-1}$ which result from asymmetric and symmetric stretching of $-\mathrm{COO}^{-}$.

Experimentally, the most sensitive technique, i.e., chemical modulated excitation (c-ME), has been applied to perform this proof. For technical details, see Section 2.2.3.

The results are presented in Figures 7 and 8. The excitation of the immobilized layer was performed by periodic exchanges of the buffer solutions ( $\mathrm{pH} 4$ and 8) in the S-cuvettes. Since the two buffer solutions are expected to have different IR spectra, the same ME was also performed in the R-cuvettes as a reference. Phase resolved spectra according to eq 3 are presented in Figure $7 \mathrm{~A}$ and $\mathrm{B}$, while Figure $8 \mathrm{~A}$ and $\mathrm{B}$ represents difference $\mathrm{ME}$ spectra of Figure $7 \mathrm{~B}$ and $\mathrm{A}$, revealing the layer response to $\mathrm{pH}$ changes. The result is unambiguous: $\mathrm{pH} 8$ results in the two expected stretching vibrations of the $-\mathrm{COO}^{-}$group, while $\mathrm{pH}$ 4 restores reversibly the protonated state of the silane layer.

For the sake of clarity, some remarks concerning ME technique should be made. (i) Demodulation was performed only in the fundamental, i.e., for $k=1$ in eq 3, (ii) At operators phase setting $\phi_{k}^{\mathrm{PSD}} \approx 82^{\circ}$, all signals vanish in the whole spectral range. According to eq 3 this means that the systems response exhibits the same phase shift $\phi_{k} \approx 82^{\circ}$ for all bands. As a consequence

(34) Usher, C. R.; Michel, A. E.; Grassian, V. H. Chem. Rev. 2003, 103, 4883-4939.

(35) Dubowski, Y.; Vieceli, J.; Tobias, D. J.; Gomez, A.; Lin A.; Nizkorodov, S. A.; McIntire, T. M.; Finlayson-Pitts, B. J. J. Phys. Chem. A 2004.

(36) Matijašević, J.; Glowacky, J.; Koker, T.; Heissler, S.; Faubel, W. and Fringeli, U. P. Langmuir, submitted.

(37) Glowacky, J.; Heissler, St.; Leiste, H.; Koker, T.; Faubel, W.; Gerdes, A. Investigation of the formation of ultrathin siloxane films on functionalized Germanium crystals by FTIR-ATR spectroscopy, International Conference on Advanced Vibrational Spectroscopy 4 (ICAVS4), 2007.

(38) Fringeli, U. P. ATR and reflectance IR spectroscopy, applications, In Encyclopedia of Spectroscopy and Spectrometry; Lindon, J. C., Tranter, G. E., Holmes, J. C., Eds.; Academic Press: San Diego, 1999; pp 58- 75. 

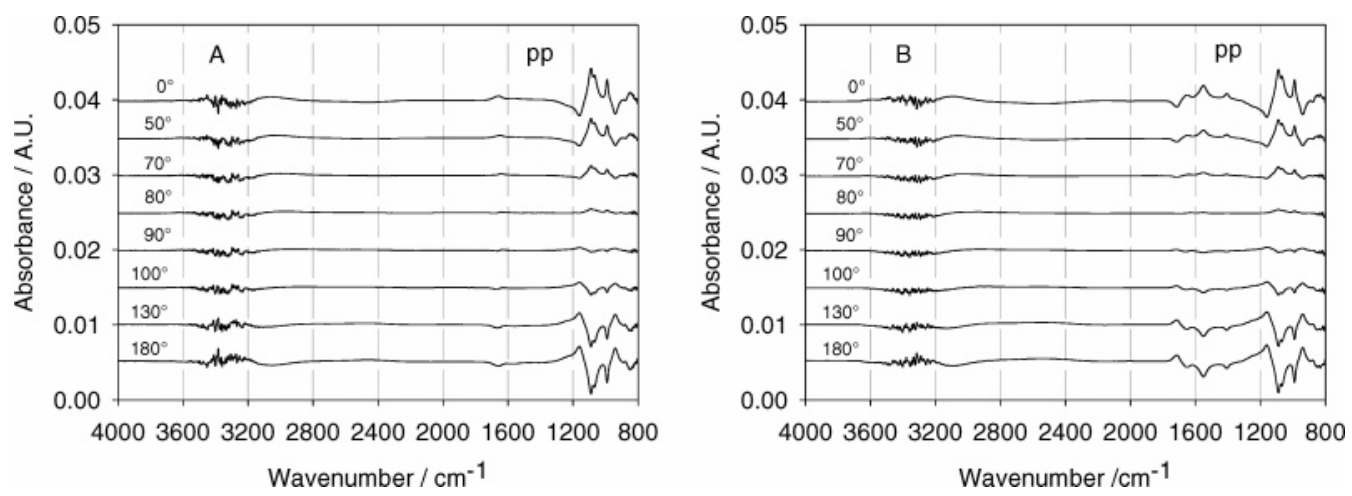

Figure 7. Parallel polarized (pp) phase-resolved absorbance spectra according to eq 3 obtained by periodic exchange of the buffer solutions (pH 4 and 8 ) in the $\mathrm{R}$ cuvettes without layer (A) and in the $\mathrm{S}$ cuvettes containing the oxidized layer (B). Modulation period $T=5 \mathrm{~min}$; Ge MIRE; $T=22^{\circ}$; angle of incidence $\theta=45^{\circ}$; number of active internal reflections $N=19.4$.
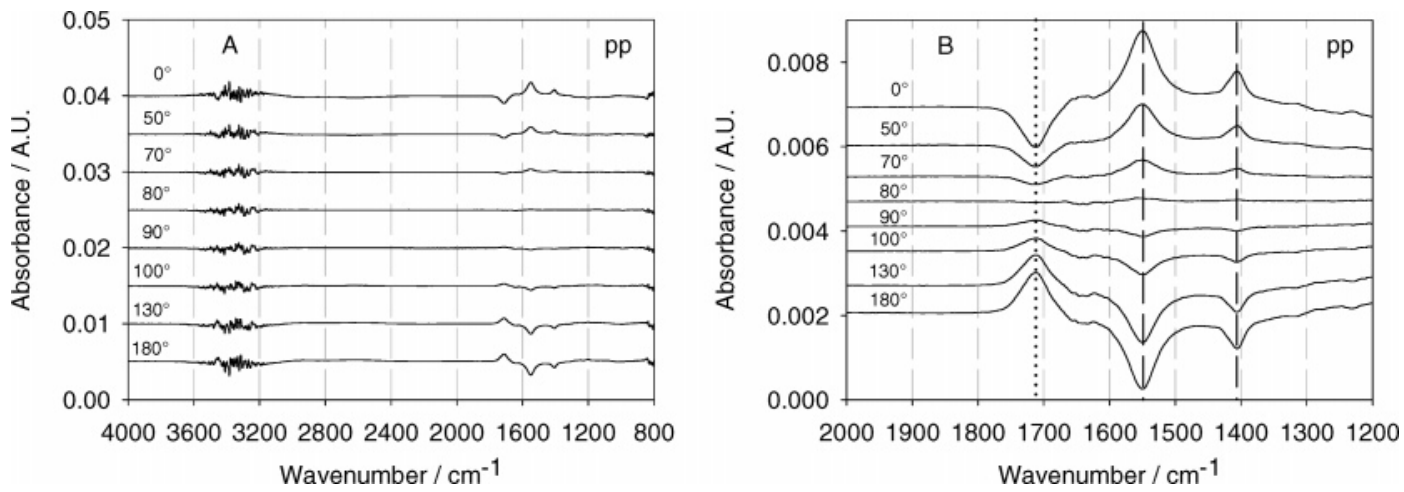

Figure 8. (A) Parallel polarized (pp) phase-resolved difference spectra between the spectra shown in Figure 7B and A, presenting the unperturbed response of the oxidized layer to the periodic $\mathrm{pH}$ change $(\mathrm{pH} 4,8)$. Spectra show a reversible change between carboxyl stretching vibration at $1718 \mathrm{~cm}^{-1}$ and carboxylate asymmetric and symmetric stretching vibrations at 1550 and $1406 \mathrm{~cm}^{-1}$, respectively. (A) Full spectral range; (B) Expanded spectral range between 2000 and $1200 \mathrm{~cm}^{-1}$. Note the exellent baseline stability of ME techniques, which is achieved by pure hardware method. Modulation period $T=5 \mathrm{~min}$; Ge MIRE; $T=22^{\circ}$; angle of incidence $\theta=45^{\circ}$; number of active internal reflections $N=19.4$.

this experiment does not reveal any phase resolution, i.e., kinetic information on protonation and deprotonation, as to be expected when considering the excitation period of $T=5 \mathrm{~min}$. The phase delay of $\phi_{k} \approx 82^{\circ}$ results from the mean time needed to exchange the solutions in the cuvettes of the SBSR flow through cell. (iii) Attention should be drawn to the high stability of background compensation. Even the very intense $\mathrm{OH}$ stretching band at 3400 $\mathrm{cm}^{-1}$ is experimentally completely compensated. Software supported baseline correction is not necessary over the whole spectral range, which documents the high reliability of ME spectra, even in the micro-absorbance range.

\section{Discussion}

This work presents in situ monitoring of the formation and quantitative analysis of an immobilized surface layer consisting predominantly of 7-carboxylsilane. The first step of silanization with $1 \%$ 7-OTCS in toluene turned out to be very critical with respect to spurious water content at any place in the reaction system. As documented by Figure 5, 7-OTCS solutions in toluene used in our experiments were dry enough in order to avoid hydrolysis of the $\mathrm{Si}-\mathrm{Cl}_{3}$ bonds in a large scale. In accordance with refs 33 and 34 , we suggest that a compact tightly bound water layer on the activated Ge surface played an important role in the silanization process. A thin water layer on Ge seems to be resistant to the presence of dry toluene; however, it was chemically removed by the reaction with $\mathrm{Si}-\mathrm{Cl}_{3}$, resulting in $\mathrm{HCl}, \mathrm{Cl}_{x}-\mathrm{Si}-(\mathrm{OH})_{y}$ and $\mathrm{Ge}-\mathrm{O}-\mathrm{Si}-(\mathrm{OH})_{z}$ at least at the beginning of the reaction. Both enable polymerization either in solution or in the immobilized layer. In our case, the latter is expected to be more dominant because 7-OTCS solution was permanently replaced by a small flow-through of $0.83 \mu \mathrm{L} / \mathrm{min}$ leading to full exchange of a cuvette volume within about one and a half minute.

A preliminary kinetic data analysis gives evidence for an accelerated initial phase followed by single-exponential growth with a time constant around $30 \mathrm{~min}$. Detailed kinetic data will be reported in a forthcoming publication. The same holds for a structural analysis during layer growth. There is some evidence for an increasing tilt angle of the hydrocarbon chains with time. However, additional low-noise, time-resolved data are required in order to get reliable results. In the final layer the mean inclination of hydrocarbon chains with respect to the normal of the layer was found to be $28.4^{\circ} \pm 0.3^{\circ}$, thus exhibiting a certain overall ordering of the assembly. Also, the mean order parameters of the $\mathrm{CH}_{2}$ groups in the hydrocarbon chains, $S_{\text {seg }}=-0.16 \pm$ 0.01 and $S_{\mathrm{mol}}=0.64 \pm 0.34$, indicate a rather good ordering of the layer. ${ }^{30}$

Quantitative analysis resulted in a surface concentration of $\Gamma_{\exp }=(6.33 \pm 0.70) \times 10^{-10} \mathrm{~mol} / \mathrm{cm}^{2}$ which corresponds to a mean layer thickness of $d=13.2 \pm 2.0 \AA$. However, on the basis of an estimation of the minimum cross-section of 7-OTCS we expect that the presented layer is at least 30\% above monolayer coverage (see Section 3.2.1). Partial multilayer formation has to be expected, also due to the fact that no $\mathrm{Si}-\mathrm{Cl}_{3}$ vibrations could be detected in an immobilized layer on a CdTe MIRE (Figure 5B and C) indicating complete hydrolization within the layer. Each $\mathrm{Si}-\mathrm{OH}$ group may be an anchor for polymerization. 
A further surface chemical step was the oxidation of the endstanding double bond of the silane layer by exposing the dried layer to an argon diluted flow of ozone. The process had led directly to the aimed carboxylic acid groups which could unambiguously be proved by concentration modulated excitation (c-ME) using buffer solutions at $\mathrm{pH} 4$ and 8. Moreover, c-ME demonstrated the power of ME techniques with respect to sensitivity, long-term stability, and selectivity. Finally, it should be mentioned that the duration of exposition to ozone, as well as the ozone concentration, has turned out to be very critical because complete oxidation of the hydrocarbon chain may occur if exposition is too long. The $\mathrm{Si}-\mathrm{O}-\mathrm{Ge}$ bonds, however, remained unaffected (spectrum not shown). In this respect, in situ monitoring turned out to be of great importance. Nevertheless, even in our approach a loss of about one-third of $\mathrm{CH}_{2}$ groups occurred, as revealed by a quantitative analysis of the final layer, see Section 3.2.3.
Acknowledgment. The authors thank to the University of Vienna for a scholarship of 6 months at the beginning of Ph.D. thesis of J. M. Prolongation of the scholarship was enabled by financial support by OPTISPEC CH-8173 Neerach, Ciba Special Chemicals CH-4000 Basel, Bruker D-76275 Ettlingen, as well as by the Institute of Biophysical Chemistry, University of Vienna, A-1090 Vienna. The autors thank Ass. Prof. Dr. Dieter Baurecht for helpful discussion and technical support with experiment. We kindly acknowledge valuable AFM investigations performed at the Karlsruhe Institute for Technical Chemistry by Jens Glowacky, Dr. Torsten Koker, Stefan Heissler, and Dr. Werner Faubel.

Supporting Information Available: Details of refs 24 and 36. This material is available free of charge via the Internet at http://pubs.acs.org.

LA703000N 